\title{
Spin- and valley-polarized transport across line defects in monolayer $\mathrm{MoS}_{2}$
}

\author{
Artem Pulkin and Oleg V. Yazyev \\ Institute of Theoretical Physics, Ecole Polytechnique Fédérale de Lausanne (EPFL), CH-1015 Lausanne, Switzerland
}

(Dated: September 26, 2018)

\begin{abstract}
We address the ballistic transmission of charge carriers across ordered line defects in monolayer transition metal dichalcogenides. Our study reveals the presence of a transport gap driven by spin-orbit interactions, spin and valley filtering, both stemming from a simple picture of spin and momentum conservation, as well as the electron-hole asymmetry of charge-carrier transmission. Electronic transport properties of experimentally observed ordered line defects in monolayer $\mathrm{MoS}_{2}$, in particular, the vacancy lines and inversion domain boundaries, are further investigated using firstprinciples Green's function methodology. Our calculations demonstrate the possibility of achieving nearly complete spin polarization of charge carriers in nanoelectronic devices based on engineered periodic line defects in monolayer transition metal dichalcogenides, thus suggesting a practical scheme for all-electric control of spin transport.
\end{abstract}

The spin and valley degrees of freedom of charge carriers are being actively considered as a means of extending the capabilities of present-day charge-based electronics. A major challenge on the way to designing practical devices for generating, manipulating and detecting spinand valley-polarized charge carriers lies in finding novel physical phenomena and suitable materials that will be utilized in such devices. Two-dimensional (2D) materials such as graphene, and more recently the transition metal dichalcogenides, appear to offer a number of valuable properties for the emerging fields of spintronics [1-4] and valleytronics [5, 6].

The family of layered transition metal dichalcogenides (TMDs) $M X_{2}(M=\mathrm{Mo}, \mathrm{W} ; X=\mathrm{S}, \mathrm{Se})$ has attracted considerable attention as prospective materials for nextgeneration electronics [7 9] and photovoltaics [10. Single layers of the $2 H$-phase TMDs are direct band gap semiconductors with valence and conduction bands located at the inequivalent $K$ and $K^{\prime}$ points of the Brillouin zone [see Fig. 1(a) for a schematic illustration of the band structure] [11 14. Single-layer TMDs lack inversion symmetry, hence a spin-orbit interaction lifts the spin degeneracy of the bands. The effect of a spin-orbit interaction is particularly pronounced in the valence band, giving rise to spin splittings of $0.15-0.46 \mathrm{eV}$ across the family of single-layer TMDs [15]. A combination of the two properties mentioned above results in an intrinsic spin-valley coupling of the hole charge carriers [16, thus making these materials an appealing choice for beyondelectronics applications. However, the potential of this novel physical phenomenon for prospective technological applications is far from being fully explored. While valley polarization of charge carriers by optical means has been recently reported [17-19], neither practical allelectric schemes for generating polarized charge carriers nor spin transport phenomena in monolayer TMDs have been demonstrated so far.

In this Rapid Communication, we propose a simple approach for generating spin- and valley-polarized charge carriers by means of transmission across periodic line defects in monolayer TMDs. Our study also reveals a number of transport phenomena in these materials, such as the suppression of hole transmission across inversion domain boundaries driven by spin-orbit splitting of the valence band. Our predictions, which are based on a simple and intuitive picture of spin and momentum conservation, are further supported by the results of first- (a)

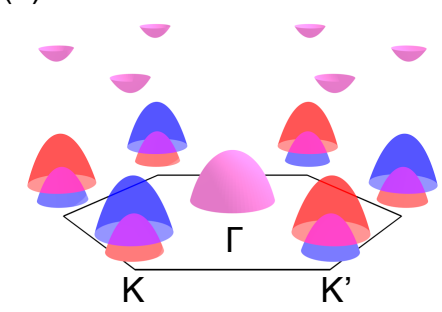

(b)

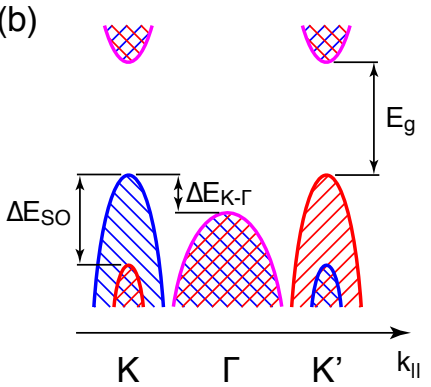

(c)

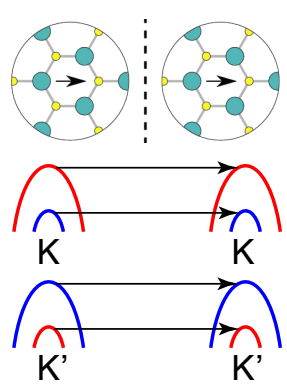

(d)

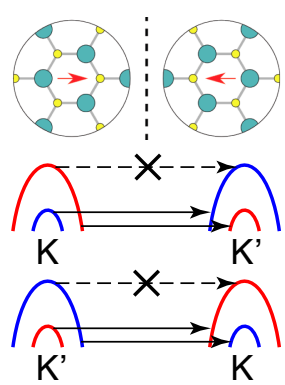

FIG. 1: (a) Schematic illustration of the electronic band structure of monolayer TMD materials showing the spin-split valence bands at points $K$ and $K^{\prime}$ of the Brillouin zone and nearly spin-degenerate valence band at $\Gamma$ and conduction bands at points $K$ and $K^{\prime}$. The spin-up and spin-down bands are color coded (red and blue). (b) Projection of the 2D band structure shown in (a) onto the direction parallel to one of the real-space lattice vectors. (c),(d) Allowed transmission channels for the hole charge carriers in the $K$ and $K^{\prime}$ valleys across line defects that preserve lattice orientation and inversion domain boundaries, respectively. The insets show the orientation of the crystalline lattice in the two domains separated by the line defect (dashed line). 
principles Green's function calculations, which reveal the quantitative aspects of spin- and valley-polarized transport across representative line defects in monolayer $\mathrm{MoS}_{2}$ that have been observed experimentally and can be engineered in a controlled manner.

We will first discuss the general phenomenology without making recourse to any particular member of the family of TMD materials or to a specific defect structure. It is, however, worth stressing that line defects in TMDs, such as grain boundaries and inversion domain boundaries (equivalent to $60^{\circ}$ grain boundaries), tend to exhibit well-ordered periodic structures $[2023]$. The periodicity vector $\mathbf{d}$ of a one-dimensional defect is defined by the commensurability condition, that is, by matching two translational vectors in the "left" and "right" domains separated by the defect 24 .

$$
\mathbf{d}=n_{\mathrm{L}} \mathbf{a}_{1, \mathrm{~L}}+m_{\mathrm{L}} \mathbf{a}_{2, \mathrm{~L}}=n_{\mathrm{R}} \mathbf{a}_{1, \mathrm{R}}+m_{\mathrm{R}} \mathbf{a}_{2, \mathrm{R}},
$$

where $\left(\mathbf{a}_{1, \mathrm{~L}}, \mathbf{a}_{2, \mathrm{~L}}\right)$ and $\left(\mathbf{a}_{1, \mathrm{R}}, \mathbf{a}_{2, \mathrm{R}}\right)$ are the lattice vectors of the "left" and "right" domains, respectively, and $\left(n_{\mathrm{L}}, m_{\mathrm{L}}\right),\left(n_{\mathrm{R}}, m_{\mathrm{R}}\right)$ are pairs of integers.

Figure 1(b) shows the band structure of a monolayer TMD projected onto the direction of momentum parallel to the defect, $k_{\|}$, when this defect is oriented along one of the lattice vectors [or, more generally, when $\left.\left(n_{\mathrm{L}(\mathrm{R})}-m_{\mathrm{L}(\mathrm{R})}\right) \bmod 3 \neq 0\right]$. In this situation, which is most often observed in experiments, the two valleys at points $K(\nu=+1)$ and $K^{\prime}(\nu=-1)$ of the Brillouin zone are separated in $k_{\|}$. Once $k_{\|}$is conserved upon transmission, two possibilities can be realized. For defects characterized by

$$
\left(n_{\mathrm{L}}-m_{\mathrm{L}}\right) \quad \bmod 3=\left(n_{\mathrm{R}}-m_{\mathrm{R}}\right) \quad \bmod 3 \neq 0,
$$

such as the structures that do not change lattice orientation [e.g., $\left.\left(n_{\mathrm{L}}, m_{\mathrm{L}}\right)=\left(n_{\mathrm{R}}, m_{\mathrm{R}}\right)=(0,1)\right]$, valley indices are conserved upon transmission. Figure 1(c) shows the allowed transport channels for hole charge carries when spin is additionally conserved upon transmission across such periodic defects. In this situation, spin and momentum conservation does not lead to transmission blocking at any charge-carrier concentration.

Figure 1(d) illustrates a different situation defined by

$$
0 \neq\left(n_{\mathrm{L}}-m_{\mathrm{L}}\right) \quad \bmod 3 \neq\left(n_{\mathrm{R}}-m_{\mathrm{R}}\right) \quad \bmod 3 \neq 0,
$$

in which the valley indices are exchanged upon crossing the defect. This scenario is realized in inversion domain boundary defects, which can be defined by the lattice vectors $\mathbf{a}_{1, \mathrm{~L}}=-\mathbf{a}_{1, \mathrm{R}}$ and $\mathbf{a}_{2, \mathrm{~L}}=-\mathbf{a}_{2, \mathrm{R}}$, and thus $\left(n_{\mathrm{L}}, m_{\mathrm{L}}\right)=(0,1)$ and $\left(n_{\mathrm{R}}, m_{\mathrm{R}}\right)=(0,-1)$, in order to satisfy the commensurability condition (1). At low concentrations of hole charge carriers, this will result in a complete suppression of transmission if both spin and momentum are conserved. At larger charge-carrier concentrations, additional allowed channels will be involved (a)

$\mathrm{d} \sim \mathrm{a}$

(b)

$d \gg a$
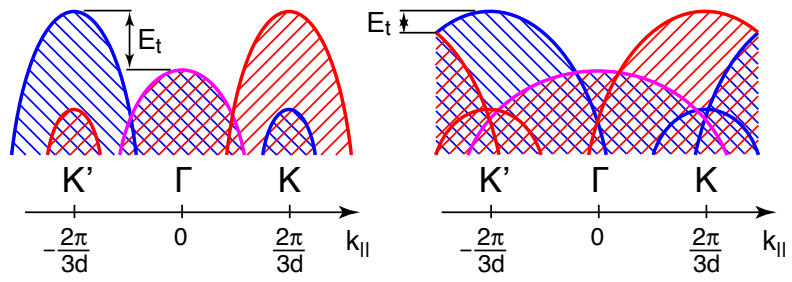

FIG. 2: Illustration of the two scenarios governing the transport gap $E_{\mathrm{t}}$ across inversion domain boundaries for the hole charge carriers is governed (a) by $\Delta E_{K-\Gamma}<\Delta E_{\mathrm{SO}}$ or (b) by the overlap between valleys $K$ and $K^{\prime}$, which is realized for sufficiently large line-defect periodicities $d$.

once the opposite-spin branches separated by spin splitting $\Delta E_{\mathrm{SO}}$ start being populated. In order to complete the picture of ballistic transmission across the inversion domain boundary defects, one needs to consider two more contributions: (i) transmission of the hole charge carriers populating the spin-degenerate valley at the $\Gamma$ point, which is separated from the valence band maximum by relatively small energies $\Delta E_{K-\Gamma}$ [see Fig. 11(b)], and (ii) the overlap of the $K$ and $K^{\prime}$ valleys along $k_{\|}$at larger $d$, as illustrated in Fig. 2. Overall, one can expect a strong suppression of the transmission of hole charge carriers across the inversion domain boundaries, with the magnitude of the effective transport gap defined by

$$
E_{\mathrm{t}}=\min \left[\Delta E_{\mathrm{SO}}, \Delta E_{\mathrm{K}-\Gamma}, E_{0} \frac{a}{d}\right],
$$

where $E_{0}=h^{2} /\left(72 m^{\star} a^{2}\right)$ is the characteristic energy of the valence band with an effective charge-carrier mass $m^{\star}$, and $a$ is the lattice constant.

The fact that the two valleys are separated in $k_{\|}$in the considered scenarios will result in discrimination of the transmitted charge carriers with respect to their valley index $\nu$ and incidence angle $\theta \in(-\pi / 2, \pi / 2)$. This effect was originally predicted 29] for a particular experimentally observed line defect in graphene [30, 31. We argue, however, that valley filtering is expected for any periodic line defect which leaves the two valleys separated in $k_{\|}$space since the following property holds for valley-resolved transmissions,

$$
T_{\nu}(\theta)=T_{\nu}\left(k_{\|}\right)=T_{-\nu}\left(-k_{\|}\right)=T_{-\nu}(-\theta) \neq T_{-\nu}(\theta),
$$

for the incidence angle $\theta$ bijectively related to momentum $k_{\|}$in the one-dimensional (1D) Brillouin zone of the defect. The corresponding valley polarization of the transmitted charge

$$
P_{\nu}(E, \theta)=\frac{T_{\nu=+1}(E, \theta)-T_{\nu=-1}(E, \theta)}{T_{\nu=+1}(E, \theta)+T_{\nu=-1}(E, \theta)}
$$

is generally nonzero for any $\theta \neq 0$. At low concentrations, the valley polarization of hole charge carriers $P_{\nu}$ is equiv- 
(a) SVL

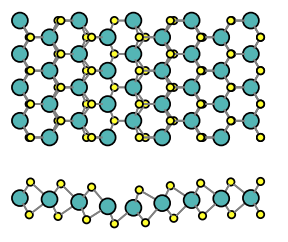

IDB1

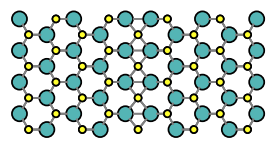

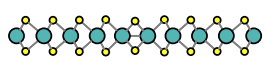

IDB2

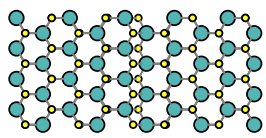

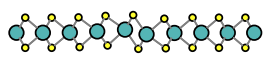

(b)

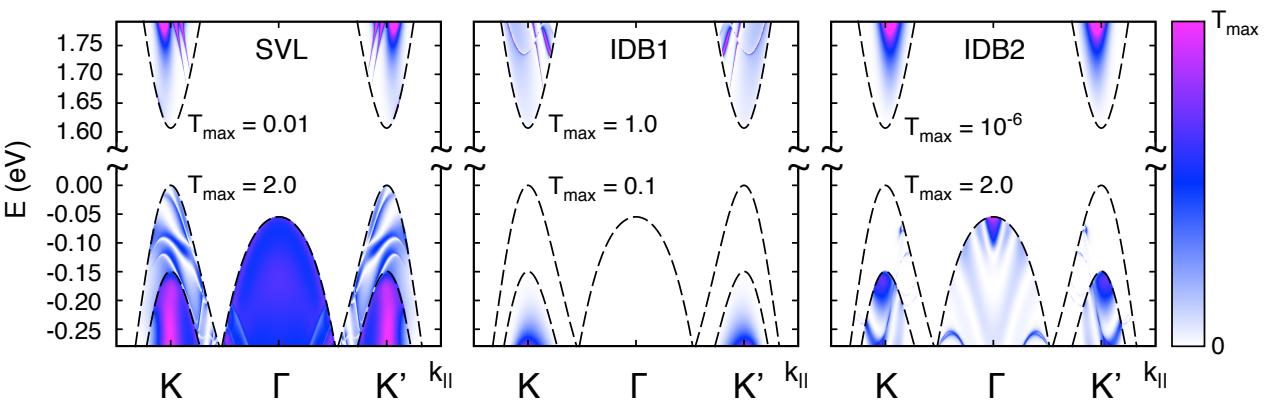

(c)

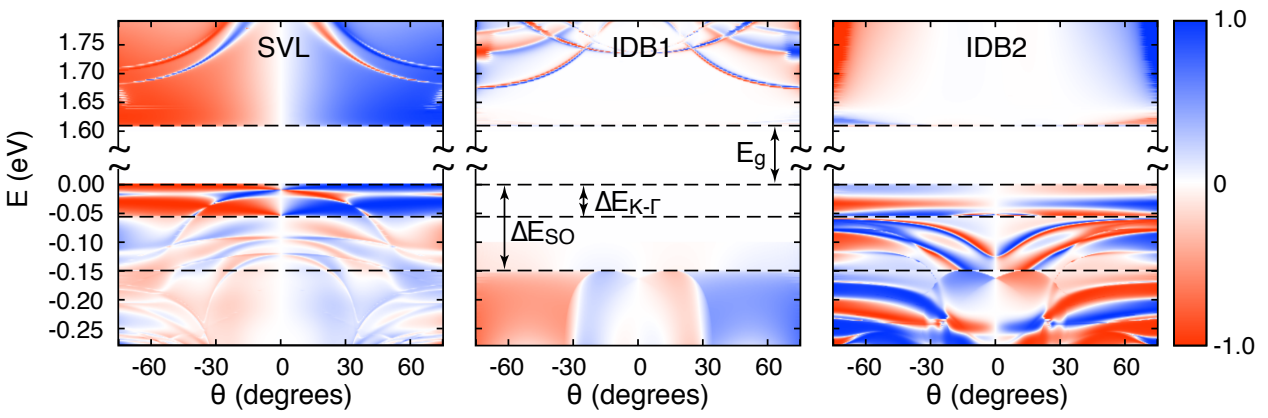

FIG. 3: (a) Top and side views of the relaxed atomic structures of periodic line defects in monolayer $\mathrm{MoS}_{2}$, the single vacancy line (SVL), and two different structures of inversion domain boundaries (IDB1 and IDB2), that have been observed experimentally [20, 25,28]. (b) Charge-carrier transmissions $T\left(E, k_{\|}\right)$across the three investigated line defects in monolayer $\mathrm{MoS}_{2}$ as a function of energy $E$ relative to the valence band maximum and momentum $k_{\|}$. The contours of the bulk bands projected onto the defect direction are shown as dashed lines. The transmissions of electron and hole charge carriers are shown on different scales defined by the upper limit $T_{\max }$. (c) Spin polarization of the transmitted charge carriers $P_{\sigma}(E, \theta)$ as a function energy $E$ and incidence angle $\theta$ calculated for the three studied line defects in monolayer $\mathrm{MoS}_{2}$. Dashed lines reflect the positions of the band edges.

alent to their spin polarization $P_{\sigma}$, due to the intrinsic spin-valley coupling in monolayer TMDs [16]. The latter property, however, is different as spin is an intrinsic property of an electron, hence spin-polarized charge carriers can be injected into other materials. The considered defects can thus be used for controlling the spin polarization of charge carriers by all-electric means without the use of magnetic materials.

In order to investigate the quantitative aspects of the predicted spin- and valley-polarized transport phenomena, we perform first-principles quantum transport simulations using the Green's function technique with spinorbit interactions accounted for by using two-component spinor wave functions in combination with fully relativistic norm-conserving pseudopotentials (for a detailed description, see the Supplemental Material 34]). Without loss of generality, we further focus our attention on monolayer $\mathrm{MoS}_{2}$ as a representative member of the monolayer TMD materials family. In our density functional theory calculations, we obtain $E_{\mathrm{g}}=1.61 \mathrm{eV}$ for the band gap. The calculated band offset between the $K$ and $\Gamma$ valence bands $\Delta E_{\mathrm{K}-\Gamma}=0.055 \mathrm{eV}$, while the spin splitting of the valence bands at $K$ points $\Delta E_{\mathrm{SO}}=0.15 \mathrm{eV}$, both in agreement with experiments and previous calculations [13, 15. The effective mass of hole charge carriers $m^{\star}=0.57 m_{e}$ corresponds to the characteristic energy
$E_{0}=0.73 \mathrm{eV}$. As an example of a periodic line defect realizing the situation shown in Fig. 1(c), we consider the single vacancy line (SVL) defects reported by Komsa et al. [25]. Two different structures of inversion domain boundaries (IDB1 and IDB2), observed in Refs. 20, 2628, were considered as examples of systems realizing the second transport scenario shown in Fig. 1(d). All three defect structures are oriented along one of the lattice vectors (often referred to as the zigzag direction) and have the smallest possible periodicity $d=a$. Importantly, the above-mentioned ordered defects can be engineered with a fair degree of control at transmission electron microscopy conditions 25 28. The atomic structures of these line defects are shown in Fig. 3(a).

Figure 3(b) shows the calculated charge-carrier transmissions $T\left(E, k_{\|}\right)$, across the investigated line defects in $\mathrm{MoS}_{2}$, as a function of energy $E$ relative to the valence band maximum and momentum $k_{\|}$parallel to the defect. One can immediately notice the predicted suppression of transmission across the inversion domain boundaries (IDB1 and IDB2) for the low-energy hole charge carriers. In monolayer $\mathrm{MoS}_{2}$, the transport gap is governed by the $K-\Gamma$ band offset rather than the spin splitting of the $K$ valley bands, that is, $E_{\mathrm{t}}=\Delta E_{\mathrm{K}-\Gamma}$ [cf. Eq. (4)]. In the case of the IDB1 defect, the transmission is strictly zero for $0>E>-E_{\mathrm{t}}$, while for the IDB2 structure a resid- 
ual transmission not exceeding $10^{-3}$ was found within the predicted transport gap. The latter is enabled by the spin-flip process due to out-of-plane bending at the defect line [Fig. 3(a)]. In general, $T\left(E, k_{\|}\right)$shows strong variations in both $E$ and $k_{\|}$, with linelike suppression typical of resonant backscattering on localized states hosted by the defects [20, 32]. A similar phenomenon was also reported for the charge-carrier transmission across a line defect in graphene 31.

Figure 3(c) shows the calculated spin polarization $P_{\sigma}(E, \theta)$ of transmitted charge carriers as a function of their energy $E$ and incidence angle $\theta$. The most prominent spin polarization is found for the SVL defect at energies $0>E>-\Delta E_{\mathrm{K}-\Gamma}$ where the hole charge carriers belong only to fully spin-polarized valleys $K$ and $K^{\prime}$. For instance, at $E=-10 \mathrm{meV}$, spin polarization achieves $P_{\sigma}= \pm 0.997$ at incidence angles $\theta= \pm 30^{\circ}$. Importantly, at these charge-carrier energies the transmissions are also high, of the order of 1 . This combination of properties identifies the optimal conditions for using ordered vacancy line defects in nanoscale spintronic devices. At $E<-\Delta E_{\mathrm{K}-\Gamma}$ the spin polarization dramatically reduces due to a large contribution to conductance of charge carriers in the spin-degenerate $\Gamma$ valley, which is also characterized by large transmissions. Interestingly, large values of $P_{\sigma}$ are also found for the electron charge carriers, although spin-orbit effects in the conduction band are generally weaker and have a complex character. In this case, transmissions are found to be about two orders of magnitude lower.

For the IDB1 defect we generally find a lower degree of $P_{\sigma}$ across the relevant range of values of $E$ and $\theta$. The dominant contribution to the transmission of holes comes from conductance channels involving $K$ and $K^{\prime}$ valleys, as indicated in Fig. 11(d). A sharp decrease of spin polarization within $\theta \approx \pm 30^{\circ}$ is due to the fact that only one of the two indicated channels is realized in this range of incidence angle values 33 . Specifically, transmission to the topmost valence band without a spin flip in this region is prohibited. The degree of spin polarization $P(E, \theta)$ of holes transmitted across IDB2 shows large variations with respect to energy $E$ and angle $\theta$, even in the energy range which corresponds to residual transmission enabled by the spin-flip process. The sign of $P_{\sigma}$ at a constant incidence angle $\theta$ changes at $E \approx-20 \mathrm{meV}$. For $E<-\Delta E_{\mathrm{K}-\Gamma}$ multiple conductance channels compete, resulting in an irregular behavior of spin polarization.

It is interesting to note the pronounced electron-hole asymmetry of the ballistic conductances in all three considered cases [Fig. 3(b)]. The calculated transmissions of the hole charge carrier across the SVL and IDB2 line defects tend to approach their maximum nominal value of 2 , which is the largest number of transmission channels within the investigated energy range. In contrast, the transmission of electron charge carriers is greatly suppressed across both defects. The opposite is true for the

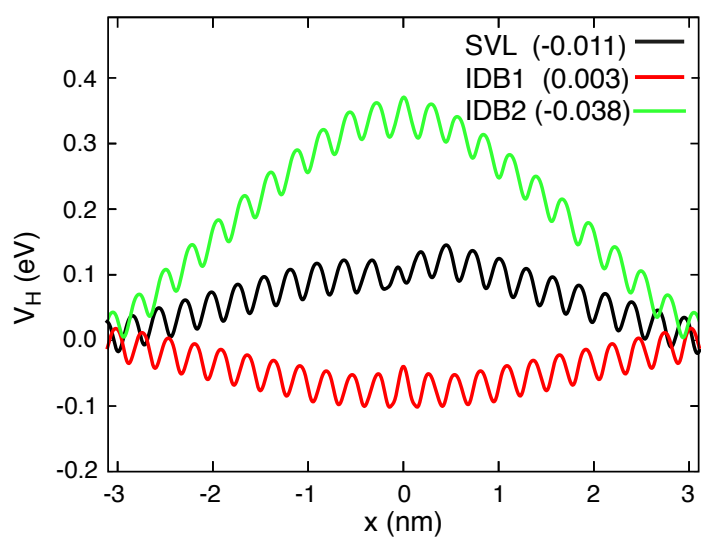

FIG. 4: Self-consistent Hartree potential $V_{\mathrm{H}}$ averaged over the planes perpendicular to the transport direction for the three line-defect models investigated in our work. The average $V_{\mathrm{H}}$ of the bulk monolayer $\mathrm{MoS}_{2}$ contacts is set to zero in all three cases. The position of the defect is at $x=0$. Calculated effective charges of defects per lattice constant length are given.

IDB1 line defect - the transmission of low-energy electron charge carriers is generally higher, which was also predicted for the same defect structure in $\mathrm{MoSe}_{2}$ [27]. We explain this behavior from the point of view of electrostatic potential bending at the defect, which can be quantified in terms of the self-consistent Hartree potential $V_{\mathrm{H}}$ within the scattering region obtained from our first-principles calculations (Fig. 4). For both the SVL and IDB2 line defects we observe upward potential bending which leads to a tunnelinglike transmission of electrons (hence their lower transmission), but not holes. Moreover, the larger height of the potential barrier in the case of IDB2 is reflected in lower transmissions of low-energy electrons in comparison with the SVL defect. The reversed behavior of charge carriers crossing the IDB1 defect is related to the downward potential bending. The sign and magnitude of potential bending is defined by an effective charge localized on the defect (cf. Fig. 4), and a competing contribution due to the head-to-head change of polarization taking place upon crossing the IDB1 and IDB2 defects, as shown in the inset to Fig. 1(d). A similar polarization discontinuity has recently been predicted to occur at the interfaces created upon selective functionalization of $2 \mathrm{D}$ materials 35]. The contribution of polarization discontinuity dominates in the case of the IDB1 structure, thus leading to a downward potential bending, while the effective large negative charge in the case of the IDB2 defect results in an upward potential bending.

In conclusion, our work reveals a number of transport phenomena in the transmission of charge carriers across ordered line defects in monolayer $\mathrm{MoS}_{2}$ stemming from a simple and intuitive picture of spin and momentum conservation combined with strong spin-orbit effects in this two-dimensional material. The results are valid for 
other members of the monolayer TMD family of materials, but one can also expect such phenomena to be observed in other semiconductors featuring strong spinorbit interactions. Our work constitutes an important step towards understanding the transport properties of realistic samples of monolayer TMDs. Furthermore, we believe it also opens an avenue towards conceptually different nanoscale devices for electronics, and their extensions, such as spintronics and valleytronics. The energy of the charge carriers in such devices operated in the ballistic regime can be controlled by means of gating, while the angle dependence can be harnessed by positioning local contacts in predefined configurations relative to the line defect 36 38]. For the purpose of confirming the predicted filtering phenomenon, coupled spin and valley polarizations of the charge carriers can be detected using ferromagnetic contacts or by optical means [17, 18, 39]. For instance, one can perform spatially resolved measurements of the circular polarization of light emitted upon electroluminescence [40] in devices containing line defects. Practical devices relying on all-electric schemes could rather use components in the valve configuration, as suggested for a graphene-based valleytronic device [31. Considering the recently demonstrated long lifetimes of spin-polarized charge carriers in monolayer TMDs [41, our work opens an avenue towards developing practical schemes for achieving all-electric control of spin transport in spintronic devices.

We thank G. Autès, F. Gargiulo, and A. Kis for discussions. This work was supported by the Swiss NSF (Grant No. PP00P2_133552) and the ERC Starting grant "TopoMat" (Grant No. 306504). First-principles calculations have been performed at the Swiss National Supercomputing Centre (CSCS) under project s515.

[1] S. A. Wolf, D. D. Awschalom, R. A. Buhrman, J. M. Daughton, S. von Molnár, M. L. Roukes, A. Y. Chtchelkanova, and D. M. Treger, Science 294, 1488 (2001).

[2] I. Žutić, J. Fabian, and S. Das Sarma, Rev. Mod. Phys. 76, 323 (2004).

[3] C. Chappert, A. Fert, and F. N. Van Dau, Nature Mater. 6, 813 (2007).

[4] A. Fert, Rev. Mod. Phys. 80, 1517 (2008).

[5] A. Rycerz, J. Tworzydło, and C. W. J. Beenakker, Nature Phys. 3, 172 (2007).

[6] D. Xiao, W. Yao, and Q. Niu, Phys. Rev. Lett. 99, 236809 (2007).

[7] B. Radisavljevic, A. Radenovic, J. Brivio, V. Giacometti, and A. Kis, Nature Nanotechnol. 6, 147 (2011).

[8] B. Radisavljevic, M. B. Whitwick, and A. Kis, ACS Nano 5, 9934 (2011).

[9] Q. H. Wang, K. Kalantar-Zadeh, A. Kis, J. N. Coleman, and M. S. Strano, Nature Nanotechnol. 7, 699 (2012).

[10] M. Bernardi, M. Palummo, and J. C. Grossman, Nano Lett. 13, 3664 (2013).
[11] A. Splendiani, L. Sun, Y. Zhang, T. Li, J. Kim, C.Y. Chim, G. Galli, and F. Wang, Nano Lett. 10, 1271 (2010).

[12] K. F. Mak, C. Lee, J. Hone, J. Shan, and T. F. Heinz, Phys. Rev. Lett. 105, 136805 (2010).

[13] W. Jin, P.-C. Yeh, N. Zaki, D. Zhang, J. T. Sadowski, A. Al-Mahboob, A. M. van der Zande, D. A. Chenet, J. I. Dadap, I. P. Herman, et al., Phys. Rev. Lett. 111, 106801 (2013).

[14] Y. Zhang, T.-R. Chang, B. Zhou, Y.-T. Cui, H. Yan, Z. Liu, F. Schmitt, J. Lee, R. Moore, Y. Chen, et al., Nature Nanotechnol. 9, 111 (2014).

[15] Z. Zhu, Y. Cheng, and U. Schwingenschlögl, Phys. Rev. B 84, 153402 (2011).

[16] D. Xiao, G.-B. Liu, W. Feng, X. Xu, and W. Yao, Phys. Rev. Lett. 108, 196802 (2012).

[17] H. Zeng, J. Dai, W. Yao, D. Xiao, and X. Cui, Nature Nanotechnol. 7, 490 (2012).

[18] K. F. Mak, K. He, J. Shan, and T. F. Heinz, Nature Nanotechnol. 7, 494 (2012).

[19] T. Cao, G. Wang, W. Han, H. Ye, C. Zhu, J. Shi, Q. Niu, P. Tan, E. Wang, B. Liu, et al., Nature Commun. 3, 887 (2012).

[20] W. Zhou, X. Zou, S. Najmaei, Z. Liu, Y. Shi, J. Kong, J. Lou, P. M. Ajayan, B. I. Yakobson, and J.-C. Idrobo, Nano Lett. 13, 2615 (2013).

[21] A. M. van der Zande, P. Y. Huang, D. A. Chenet, T. C. Berkelbach, Y. You, G.-H. Lee, T. F. Heinz, D. R. Reichman, D. A. Muller, and J. C. Hone, Nature Mater. 12, 554 (2013).

[22] S. Najmaei, Z. Liu, W. Zhou, X. Zou, G. Shi, S. Lei, B. I. Yakobson, J.-C. Idrobo, P. M. Ajayan, and J. Lou, Nature Mater. 12, 754 (2013).

[23] O. V. Yazyev and Y. P. Chen, Nature Nanotechnol. 9, 755 (2014).

[24] O. V. Yazyev and S. G. Louie, Nature Mater. 9, 806 (2010).

[25] H.-P. Komsa, S. Kurasch, O. Lehtinen, U. Kaiser, and A. V. Krasheninnikov, Phys. Rev. B 88, 035301 (2013).

[26] Y.-C. Lin, D. O. Dumcenco, Y.-S. Huang, and K. Suenaga, Nature Nanotechnol. 9, 391 (2014).

[27] O. Lehtinen, H.-P. Komsa, A. Pulkin, M. B. Whitwick, M.-W. Chen, T. Lehnert, M. J. Mohn, O. V. Yazyev, A. Kis, U. Kaiser, et al., ACS Nano 9, 3274 (2015).

[28] J. Lin, S. T. Pantelides, and W. Zhou, ACS Nano 9, 5189 (2015).

[29] D. Gunlycke and C. T. White, Phys. Rev. Lett. 106, 136806 (2011).

[30] J. Lahiri, Y. Lin, P. Bozkurt, I. I. Oleynik, and M. Batzill, Nature Nanotechnol. 5, 326 (2010).

[31] J. H. Chen, G. Autès, N. Alem, F. Gargiulo, A. Gautam, M. Linck, C. Kisielowski, O. V. Yazyev, S. G. Louie, and A. Zettl, Phys. Rev. B 89, 121407 (2014).

[32] X. Zou, Y. Liu, and B. I. Yakobson, Nano Lett. 13, 253 (2013).

[33] T. Habe and M. Koshino, Phys. Rev. B 91, 201407 (2015).

[34] See Supplemental Material at http://link.aps.org/supplemental/... for the detailed description of computational methodology.

[35] M. Gibertini, G. Pizzi, and N. Marzari, Nature Commun. 5, 5157 (2014).

[36] Q. Yu, L. A. Jauregui, W. Wu, R. Colby, J. Tian, Z. Su, H. Cao, Z. Liu, D. Pandey, D. Wei, T. F. Chung, P. Peng, 
N. P. Guisinger, E. A. Stach, J. Bao, S.-S. Pei, and Y. P. Chen, Nature Mater. 10, 443 (2011).

[37] A. W. Tsen, L. Brown, M. P. Levendorf, F. Ghahari, P. Y. Huang, R. W. Havener, C. S. Ruiz-Vargas, D. A. Muller, P. Kim, and J. Park, Science 336, 1143 (2012).

[38] G.-H. Lee, G.-H. Park, and H.-J. Lee, Nature Phys., doi:10.1038/nphys3460 (2015).

[39] W.-Y. Shan, J. Zhou, and D. Xiao, Phys. Rev. B 91,
$035402(2015)$.

[40] J. S. Ross, P. Klement, A. M. Jones, N. J. Ghimire, J. Yan, D. G. Mandrus, T. Taniguchi, K. Watanabe, K. Kitamura, W. Yao, D. H. Cobden, and X. Xu, Nature Nanotechnol. 9, 268 (2014).

[41] L. Yang, N. A. Sinitsyn, W. Chen, J. Yuan, J. Zhang, J. Lou, and S. A. Crooker, Nature Phys. 11, 830 (2015). 